\title{
Investigation and Research on the Ordinary University High Level Track and Field Sports Teams in Sichuan Province
}

\author{
Dawei $\mathrm{Li}^{1}$, Bin Wang ${ }^{2}$ \\ College of Physics Education, Neijiang Normal University, Neijiang, Sichuan, China
}

\begin{abstract}
: by using the method of literature, questionnaire and mathematical statistics and comparative analysis and research method of sichuan university high level survey of the Track and field team, sichuan university high level Track and field team and the analysis of existing problems and research, in order to sichuan university of high level Track and field teams development to provide the reference.
\end{abstract}

Keywords: sichuan; Ordinary university; The high leve Track and field team; research

\section{Introduction}

Sichuan province is one of the earliest provinces trying to do high level sports teams in China. In the past 20 years, it has made great achievement, while also existed many problems, for example: imperfect competition system, serious lack of excellent students, lack of funds, low efficiency of coaches, "learning and training" contradiction, etc. therefore, this paper made a further analysis on project layout, recruiting students, management system, competition rules, financing Investment, training effect and coaches of university track-and-field team in Sichuan province. Trying to find out problems and providing provides the theory basis for the positive development of the track team and the advance of sports level, offering a force for functional department policies and for the healthy development of track and field sports in universities of Sichuan province. It has important theoretical and practical significance on improving the whole quality of sports talent, training excellent sports talents, promoting the development of competitive sports.

\section{Research object and research me- thods}

\subsection{Research object}

This paper takes 131 high level athletes, including male 67, female 64 as the research object, who are from 9 universities in Sichuan, and they are Sichuan University(SU), University of Electronic Technology(UET), Southwest Jiaotong University (SWJTU), Chengdu University of Traditional Chinese Medicine(CUTCM), Xihua University(XU), Xihua Normal University(XNU) and Southwest Oil University Institute of Cheng$\mathrm{du}$ (SOUIC). It also makes an investigation about experts and coaches in the fields of track and sports training project and university. It contains investigation $20 \mathrm{ex}-$ perts, 16 male and 4 female; investigation coaches 31 , 23 male and 8 female.

\subsection{Research Methods}

2.2.1 Literature Study

2.2.2 Expert Interview

2.2.3 investigation method

2.2.3.1 Questionnaire

Respectively issue questionnaires survey to coaches and athletes of high level track and field in 3 universities, such as Sichuan University, University of Electronic Technology, and Southwest Jiaotong University (SWJTU).

2.2.3.1.1 The Design of the Questionnaire and Validity of Inspection

\subsection{The Design of the Questionnaire}

Before making questionnaire, read lots of books about social survey, research methods and athletic sports. According to the research content and purpose, interviews with experts, and on the basis of research methods, in strict accordance with the basic requirements of the questionnaire, adopt the suggestion from supervisor and experts to design coaches and athletes questionnaire, and finally carry on the questionnaire validity and reliability test, to determine the feasibility of the questionnaire. Combine close and open method to design the athletes, coaches and experts questionnaire.

Inspect the questionnaire validity in the way of agreement by experts. After two rounds to delete, supplemented and adjustment, the questionnaire finally complies with the requirements of the validity of the test. 2.2.3.1.1.2 Questionnaire Reliability Testing

The reliability of the questionnaire inspection uses two tests methods, respectively test the subjects of experts, coaches and athletes then test again, the time interval of two measures for 15 days. Calculate two questionnaires correlation coefficient by SPSS, experts questionnaire $\mathrm{R}=0.892, \mathrm{p}<0.01$, coaches questionnaire $\mathrm{R}=0.873, \mathrm{P}<0.01$, athletes questionnaire $\mathrm{R}=0.854$, 
$\mathrm{p}<0.01$. To illustrate the high reliability, the reliability of three copies agrees with requirements.

\subsection{Issue and recycling}

From the mid of April to Augest in 2011, issue questionnaire by onsite and delivery. Please look at the following table. This investigation of the questionnaire recovery is more than $70 \%$, so the questionnaire can meet the needs of the study.

Table 1 Issue and recycling

Type issue recycle valid recovery effective rate

\begin{tabular}{lllllll}
\hline Athletes & 140 & 134 & 131 & $95.7 \%$ & $97.7 \%$ \\
\hline coaches & 35 & 33 & 31 & $94.2 \%$ & $93.9 \%$ \\
Experts & 20 & 20 & 20 & $100 \%$ & $100 \%$
\end{tabular}

\subsubsection{Expert Interview}

According to research results to write interview outline, then please experts answer related questions by written or oral.

2.2.5 Mathematical Statistics Method

Analyse statistical data by SPSS.

\subsubsection{Logical Reasoning}

Carefully compare, analyse, summarize and expound the researching view by logical knowledge and method.

\section{Results and Analysis}

3.1 External Factors Analysis on the Development of High Level Track and Field Team in Ordinary University of Sichuan province

3.1.1 The Project Layout of University High Level Sports Teams in Sichuan

The project layout in 9 ordinary universities of high level sports teams is traditional items: track, swimming, basketball, also includes Go, KongFu and Taekwondo.

table2 Distribution table of ordinary university high level sports teams

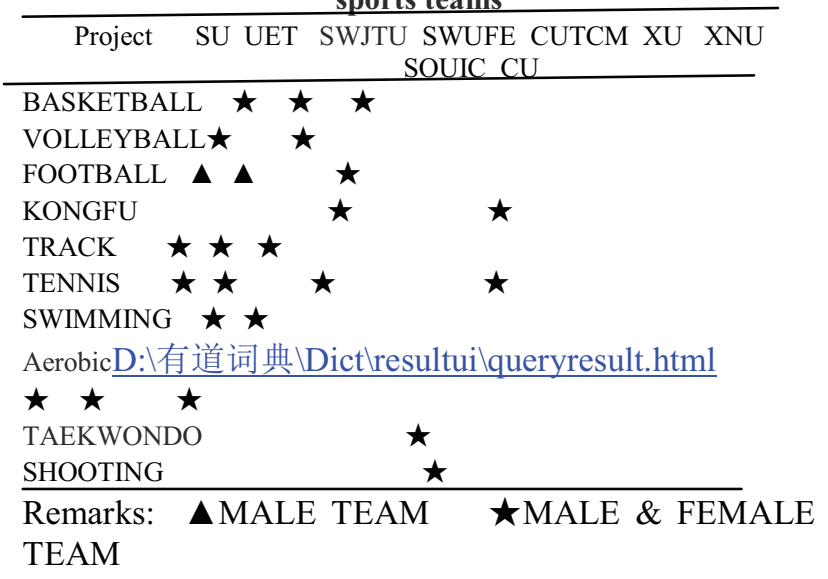

3.2 Internal Factors Analysis on the Development of High Level Track and Field Team in Ordinary Uni- versity of Sichuan province 3.2.1 Team Mode of University High Level Track Team in Sichaun

Table3 team mode of high level track sports team

\begin{tabular}{cccc}
\hline$(\mathrm{N}=31)$ & frequency & $\%$ & order \\
\hline school & 19 & $61.3 \%$ & 1 \\
province\&school & 9 & $29.0 \%$ & 2 \\
province team school-run & 3 & $9.7 \%$ & 3
\end{tabular}

According to table 3: school takes up $61.3 \%$, province \& school is $29.0 \%$, and province team school-run takes up $9.7 \%$. At present, the ordinary university high level sports teams in Sichuan province form proper motion team organized with characteristics of sports. Province team school-run, Sports authority distributes funds directly to college, realizing the real meaning management of high level athletes in the school. Universities and province professional leagues joint team together, schools are responsible for athletes' culture study and students' identity management, professional leagues are responsible for the athlete's training, schools take part in the contest, when the professional leagues have duty,they are on behalf of the professional leagues to take part in the game.

Table 4 School athletics team training system $(\mathrm{N}=31)$

\begin{tabular}{lllll}
\multicolumn{3}{c}{ frequency } & $\%$ & order \\
\hline one-stop training system & 5 & $16.7 \%$ & 3 \\
Introduction system & 4 & $13.3 \%$ & 4 & \\
University-centered system & 14 & $46.7 \%$ & 1 \\
School association system 7 & $23.3 \%$ & 2 & \\
\hline
\end{tabular}

Above all, now University-centered system takes up $46.7 \%$, School association system $23.3 \%$, one-stop training system $16.7 \%$, Introduction system $13.3 \%$.

\subsubsection{Athletes Sources}

Table 5 identity before entering the college

\begin{tabular}{|c|c|c|c|}
\hline \multicolumn{2}{|c|}{$(\mathrm{N}=131)$} & frequency $\%$ & order \\
\hline High school students & 76 & $57.6 \%$ & 1 \\
\hline Sports students & 38 & $29.0 \%$ & 2 \\
\hline Athletes in Sports Team & 7 & $5.3 \%$ & 4 \\
\hline Retired Athletes & 10 & $7.6 \%$ & 3 \\
\hline
\end{tabular}

From table 5: high school students 57.6\%; sports students $29.0 \%$; retired Athletes 7.6\%; Athletes in sports team only 5.3\%. Sports level in College athletes generally is not high. The large proportion of students is from ordinary schools, and this part of players only has little importantce to improve college athletic sports.

3.2.3 Learning and Training Contradiction of Athletes in High Level Track and Field Team

\begin{tabular}{ccccc} 
Table 6 daily training influents study \\
\hline (N=131) & frequency & $\%$ & order \\
\hline No influence & 32 & $24.4 \%$ & 2 \\
Some influence & 79 & $60.3 \%$ & 1 \\
Much influence & 16 & $12.2 \%$ & 3 \\
Serious influence & 4 & $3.0 \%$ & 4 \\
\hline
\end{tabular}

Apparently no influence is $24.4 \%$; while some influence, much influence and serious influence is $60.3 \%$, 
$12.2 \%$ and $3.0 \%$, which shows athletes are influenced by study. High level athletes spend much energy and time on training and contests, at the same time, they also should finish study test, it is quite difficult for them.

Table 7 how to deal with games and courses

\begin{tabular}{lcccc}
\hline \multicolumn{1}{c}{$(\mathrm{N}=131)$} & \multicolumn{2}{c}{ frequency } & $\%$ & order \\
\hline Exemption & 9 & $6.9 \%$ & 4 \\
Deferment examination & 48 & $36.6 \%$ & 2 \\
Not included in the performance 11 & $8.4 \%$ & 3 \\
Tests give bonus & 55 & $42.0 \%$ & 1 \\
Others & 8 & $6.1 \%$ & 5 \\
\hline
\end{tabular}

As you know, "tests give bonus" and deferment examination is relatively $42.0 \%$ and $36.6 \%$, the forst two. In the survey, most of universities take measures reduce scores or some requirements in athletes' study, such they can graduate.

Table 8 How to solve the contradictions $(\mathrm{N}=31)$ frequency $\%$ order

Reduce the requirement of culture course $20 \quad 64.5 \% 2$

Holiday (including weekends) make up a missed lesson $\quad 13.2 \% \quad 4$

Extended learning time

$13 \quad 41.9 \% \quad 3$

Reduces The Training Time

$1 \quad 3.2 \% \quad 4$

Get scores by training \& contests, not taking nondegree courses others

$26 \quad 83.8 \% \quad 1$

\begin{tabular}{lll}
1 & $3.2 \%$ & 4 \\
\hline
\end{tabular}

In table 8 , "get scores by training \& contests, not taking nondegree courses" and "reduce the requirement of culture course" is $83.8 \%$ and $64.5 \%$, taking the first two, the reason is sports training and culture education have different rules. Excellent athletes need a lot of time to train; they can't step by step as the general cultural courses of study. If you want to change this kind of situation, you need to deeper discussion from the system, and further open and introduce new ways.

3.2.4 Training Time of Athletes in High Level Track and Field Team

Table 9 traing time

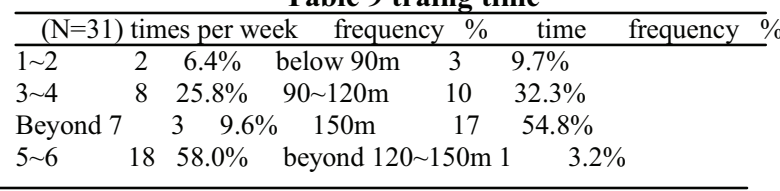

In table 9: "semester training(not including holidays) and whole year training(including holidays)" is $51.1 \%$ and $32.8 \%$, which shows the plan of high level track and field team in Sichuan is very good. In addition, "5 6

(time/week) and $90 \sim 120 \mathrm{~m}$ "is $58.0 \%$ and $54.8 \%$, and is shows training time in most universities is valuable, the detailed training plan is arranged by each university. Now the university training has gradually entered the regularization, and its measure standard only stays on training time.

3.3. Coaches' Construction of High Level Track and Field Team

Table 10 the proportion of men and women

\begin{tabular}{lcc}
\hline$(\mathrm{N}=31)$ & frequency & $\%$ \\
\hline Men & 23 & $74.2 \%$ \\
Women & 8 & $25.8 \%$ \\
\hline
\end{tabular}

At present, the proportion of coaches is large, men coaches have $23,74.2 \%$, and women coaches have 8 , $25.8 \%$, which has some relationship with coaches' working reason.

3.3.1 The Structure of Coaches

Table 11 working situation

\begin{tabular}{lcc}
\hline $\mathrm{N}=31)$ & frequency $\%$ \\
\hline Full-time & 6 & $19.4 \%$ \\
Part-time & 25 & $80.6 \%$ \\
\hline
\end{tabular}

Part-time coaches accounts for 80.6\%; Full-time accounts for $19.4 \%$. Visibly, in high level track and field teams, coaches are mostly physics teachers, full-time coaches are not many. Part-time coaches not only finish teaching task, but also serve as the other school sports work. Some coaches even undertake administrative work.

3.3.2. The coaches' age and title structure

\begin{tabular}{lccc} 
& \multicolumn{3}{c}{ Table 12 title } \\
\hline & $(\mathrm{N}=31)$ & frequency & $\%$ \\
\hline Instructor & 7 & $22.6 \%$ &
\end{tabular}

app:ds:associate app:ds:professor $\quad 20 \quad 64.5 \%$

\begin{tabular}{lll} 
Professor & 4 & $12.9 \%$ \\
\hline
\end{tabular}

Coaches' title structure reports their creativity. At present, $64.5 \%$ is associate professor, the first, associate professor of coaches have richer teaching experience and the theory training. At present, associate professor title is given priority in high level track and field team, the coach's title structure is reasonable.

3.3.3. The Coaches' Education and Experience

Table 13 education background

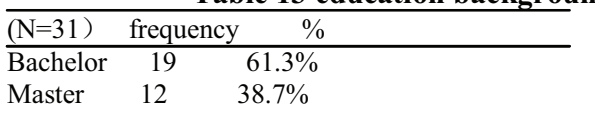

The coaches' education can reflect whether the coaches can use of the knowledge of the multidisciplinary to guide the training and competition. At present, coaches' education in high level track and field teams mostly is in the bachelor degree $(61.3 \%)$, the master degree is little $(38.7 \%)$, which has certain gap with the requirement of the ministry of education, and it puts forward to 2005, the proportion of university teachers with master degrees, the teaching research universities 
reach above $80 \%$; general universities reaches more than $60 \%$.

3.3.4. Factors Influent the Coaches Improve

Table 14 factors influent training

\begin{tabular}{lccccc}
\hline \multicolumn{4}{c}{$(\mathrm{N}=31)$} & \multicolumn{4}{c}{ frequency } & $\%$ & order \\
\hline Coaches evaluation system & 7 & $22.5 \%$ & 8 & \\
Coaches of full-time & 12 & $38.7 \%$ & 4 & \\
Education and title & 3 & $9.6 \%$ & 9 & \\
Dedication to work & 10 & $32.2 \%$ & 5 & & \\
Teaching time and experience & 8 & $25.8 \%$ & 6 & \\
Sports experience & 8 & $25.8 \%$ & 6 & \\
Leader supports & 14 & $45.1 \%$ & 3 & \\
Train funds & 19 & $61.2 \%$ & 1 & \\
Train time & 15 & $48.3 \%$ & 2 & \\
\end{tabular}

According to the results of the survey, firstly, "train funds" $61.2 \%$, shortage of funds is a common problems, to some extent, it affects sustainable development of training work in Sichuan province.

3.3.5. Practice facilities and other basic security

Table 15 Training ground and equipment can meet the need

\begin{tabular}{llll}
\hline \multicolumn{3}{c}{ frequency } & $\%$ order \\
\hline $\mathrm{N}=131)$ & \multicolumn{1}{c}{ need } \\
\hline Fully meet the need & 27 & $20.6 \%$ & 2 \\
Basically meet the need 93 & $71.0 \%$ & 1 \\
Can't meet the need 11 & $8.4 \%$ & 3 \\
\hline
\end{tabular}

$71.0 \%$ of ground and equipment can meet the need, which shows most universities can meet the training need in ground and equipment.

3.4 Development Countermeasures of Universities High Level Track and Field Team in Sichuan Province

3.4.1 People-oriented, overall development, practically and effectively solve the contradictions

University, as the base for training personnel, on the one hand, develops sports and education, has the advantages of overall development personnel training. Currently in the cultivation of college high level athletes, the contradictions between learning and training are more apparent. The present universities sports teams should strengthen athletes' supervision and management on study, and the credit system management. According to the task of training, athletes can choose the appropriate course; such there may be more comfortable time to ensure learning and training. Most universities for outstanding students in competition give part of the elective credits to encourage college athletes to learn. To strengthen the connection and communication between each colleges, and clear accountability, build an environment for graduate college athletes. Taking scientific method in the training, at the same time to think that this is in line with the concept, maximize the ease study and training, outstanding talents with all-round development in training goal.

3.4.2 Broaden the channels of recruit students; make great efforts to build a dragon training system
Our country should make efforts to guide college high level sport teams to enroll more excellent sports reserve talented person from all kinds of traditional sports school and sports characteristics school. China's "one-stop" training system is an effective way to train high level athletes; in the recruitment of students we should learn effective experience, in the policy to give outstanding athletes some priority. For a special performance athletes direct an exemption to school. "Onestop" training mode has obvious advantages: first, systematic learning, student athlete uninterrupted attend study which help students to master the knowledge. "One-stop" training mode secures the training efficiency of the high level athletes. Second, the student athletes accept systematic training from the birth which lay good sports foundation, and create the possibility to obtain higher levels later. Finally, a dragon pattern can fully mobilize students' learning interest and form a good habit of studying, and do the all-round development of morality. So building a "one-stop" athletes training linkage system accelerates cultivation of sports reserve talented person and broaden the main channel of amateur training.

3.4.3 Promote the combination of sports and education; create a high level coach team

The coach team of high quality is the key of improving sports training level, excellent coaches is undoubtedly the powerful guarantee of athletes achieving good results. In the coaches training, ministry of sports strengthens the cultivation of physics teacher in university. Through holding sports coaches post training to systematic train college sports trainers, improve the coaches' study and application of advanced training method and idea. And also improve the ability to analyze and solve problems, improve the ability of scientific research and management, develop the coaches' spirit of innovation and initiation. To continuously introduce and train of highly educated coaches, improve coaches' level of scientific research ability, improve the structure of the coaches' team, construct a complete set of scientific training program. At the same time, organize college coaches go abroad or to study advanced management experience and training experience and innovation concept, and constantly improve the coaches' ability.

3.4.4 Strengthen the application of scientific research method in sports training

In the university's track and field training, diagnosis and analysis of action and training methods is general lack, the existing training methods and means still focus on coaches' experience, university's science and technology ability doesn't sufficient play in the process of training, college sports training should continuously improve the content of science and technology and innovation, and apply the advanced technology and the concept into athletes' practice, effective feedback athletes common physical stamina and technical training 
indexes, and necessarily monitor athletes' physiological and biochemical indexes, the coaches not only depend on their own teaching experience for judgment, also on the power of science and technology to monitor the athlete's body. At present, our province most college team is not his own research team and laboratory, and has a large gap comparing domestic or international teams. University leaders give attention, and play the university scientific research ability, provide the necessary support, the university sports level, which is the development task of high level track and field teams.

3.4.5 Increase the funds investment, advance industrialization process of college sports

The lack of training funds is a common phenomenon of high level sports teams, lack of money has also been restricted the development of high level sport teams; fund is the material basis of high level sport teams. On the one hand, education funds used to education are very limited, college sport must change operation pattern under the planning economic system, and continuously through providing high levels competitive sports and college sports venues service paid open, meet the demand of the sports culture. In our province, university's track and field high level sports teams dig deeper in their economic value, walk into the market, develop their own sports brand value, and get right to speak, and actively seek social sponsorship, further promote the sports industry commercialization.

3.4.6 Increase the number of competitions; improve the athlete's actual combat sports level

Survey suggests: Sichuan province of high level track team each year besides taking part in a sports meeting in the school, every two years or four years attends to a province sports meeting, and these games level is not high, the aim is to make over, not reflect a good results.

The truth is also the scope and opportunity of contests for college track and field athletes is very few, and the motivation is to solve their own credit problem or graduation. The shortcoming of the sports and competition ability is generally one of the disadvantages. Various universities should make full use of games to promote the development of high level sport teams. Therefore, the department of education and sports should actively create a condition, increase the chance to play.

3.4.7 University high level sports teams leagues the professional, which may be a realistic way

The present training level of track team of universities high level is far not to compete in the international game, to be a high level track team, jointing the professional leagues is also a way out. China's competitive sports reserve talented person training forms "amateursports team-the professional leagues" training system. In this system, our country cultivates a large number of coaches, management personnel, research personnel and professional sports talents, invests a lot of money for improving the construction of sports facilities and training conditions. Sports system currently almost holds all the advantages of talent, scientific research, and capital, with the success of the Olympic Games, the gap is inevitable bigger and bigger. After the expansion of advance education, the fund is more scarce, used to run high level sports teams funds little and little, which lead to the gap between education systems and competitive sports system is big. In research that most coaches think key of college high level sport teams' level is not high, this is because the students' quality is not high. In our country sports training system, sports department had been mastering the rights of sports selecting talents and the competition. Based on the above reasons, team mode of universities and professional leagues do joint, though for a long time, it does not form, but the development direction of college high level sports teams jointing the professional leagues will gradually become the main mode.

\section{Conclusion}

Conclusion: (1) management regulations of high level athletes in university are not scientific, some athletes do not adapt, therefore, management regulations need to be improved. (2) the number of game experience is very short, low training motivation influence athletes to raise the level; (3) lack of learning time, training fatigue, no interest in study are the important factors on high level track and field athletes' study; (4) the universities physical teachers mainly consist of high level track team coaches, full-time coaches are less; (5) the lack of funds for training high level track and field athletes is the common problem. (6) Scientific training and research in high level training should be raised.

Suggestions: (1) Broaden the channels of recruit students; make great efforts to build a dragon training system; (2) People-oriented, overall development, practically and effectively solve the contradictions;

(3) Increase the number of competitions; improve the athlete's actual combat sports level; (4) Promote the combination of sports and education; create a high level coach team; (5) Increase the funds investment, advance industrialization process of college sports; Give full play to the advantages of university resources, interdisciplinary research, improvement of scientific training; Strengthen cooperation between sports colleges scientific research units of sports. (7) Properly deal with the contradiction between culture study and sports training; Implement separation of training and teaching to ensure development of competition and group coordination. (8) Learn foreign institutes experience on high level sport teams, make efforts to increase competition opportunities, improve the athletes' sports level.

\section{REFERENCES}


[1] Fang Qi, Liu Yunchao, the construction of a new vision on "combination of sport and education" of the ordinary university high level sports teams [J]. Journal of Capital Sports College 2008, (3) : 14-15

[2] Dai Qian. Twenty years of education in college, multidimensional scrutiny on education and sports [J]. Journal of Changchun Normal University. 2011 , (4) : 19-20

[3] Hu Xiaoming. The new road on exploring cultivation of competitive sports talents from "the combination of education and sports" to "sharing sports" [J].Sports Science. 2011, (6) : 14-15

[4] Zeng Ji. Study on the construction of "the combination of education and sports"coaches [J].Journal of ministry sports continued college. 2011(1):25
[5] Tan Hua. Several theoretic questions about sports sustainable development.[J].Sports Magazine , 2000, (5) : 13-18

[6] Yu Zhonggan, Liu Zhimin, Ding Haiyong. The situation and existing problems on competitive sports sustainable development. [J].Journal of Shanghai Sports College, 2000, (5) : 8-11

[7] Liu Haiyuan, The development of university competitive sports needs strategical study. [J]. Journal of Xian Sports College, 2003, (1) : 34-36

[8] Zhang Chunhua, Thoughts of external factors on competitive sports. [J]. Journal of Wuhan Sports college, 2000, (1) : $1-5$ 